\title{
Composiçào isotópica da água de chuva e da água do solo em floresta amazônica do tipo terra firme, regiảo de Manaus (*)
} \author{
Paulo Rodolfo Leopoldo ('); Eiichi Matsui ( ${ }^{2}$ ); Eneas Salati ( ${ }^{2}$ ); Wolfram Franken ( ${ }^{3}$ ); Maria de Nazaré
Góes Ribeiro ( ${ }^{3}$ )
}

\section{Resumo}

Observou-se os valores de $\delta^{2} \mathrm{H}$ e $\delta^{18} \mathrm{O}$ da água de chuva e suas respectivas repartições, incluindo-se a água do solo até a profundidade de $1,20 \mathrm{~m}$. O trabalho foi realizado na Bacia Modelo, em área experimental próxima ao $\mathrm{Km} 14$ da estrada ZF-2. Os resultados obtidos mostraram que os conteúdos isotópicos da água de chuva variam com a estação do ano, sendo mais rica no período que se inicia em fins de abril até início de novembro. Para a água do igarapé Tarumã-Açu essa variação não foi observada, indicando uma mistura de águas e um armazenamento pelo solo de grandes quantidades de água.

\section{INTRODUÇÃo}

Os isótopos estáveis, ${ }^{2} \mathrm{H}$ e ${ }^{18} \mathrm{O}$, têm fornecido indicações quanto a origem, a circulação e as misturas das águas dando, desse modo, informações básicas quanto ao seu comportamento.

Matsui et al. (1972 e 1976) puderam aplicar essa técnica na determinação das descargas relativas dos rios Negro e Solimões, enquanto que Reis et al. (1977) utilizaram-se de variações das concentrações de ${ }^{2} \mathrm{H} \in{ }^{18} \mathrm{O}$ na caracterização das águas da ilha Marajó.

O tempo de residência da água de chuva em algumas bacias hidrográficas da Região Amazônica foi estudado por Gonçalves (1979), através das observações dos valores de $\delta^{2} \mathrm{H}$ e $\delta^{18} \mathrm{O}$ em amostras de águas de chuva e rios.

Salati et al. (1979) verificaram que o conteúdo de $\delta^{18} \mathrm{O}$ das precipitações na Bacia Amazônica é caracterizado por um gradiente de $0,75 \times 10^{-3} \% / \mathrm{km}$ em conseqüência da umida- de reevaporada. Sequndo esses autores cerca de $44 \%$ da umidade deixa a bacia na forma de vapor, sendo a quantidade restante, recirculada na própria bacia.

Além desses trabalhos citam-se também aqueles desenvolvidos por Dall'Olio (1976), Dal!'Olio et al. (1979) e Salati et al. (1978), que so prestaram ao estudo do ciclo hidrológico da Bacia Amazônica através da técnica isotópica.

O aspecto abordado trata da observação dos conteúdos isotópicos da água de chuva e relações com as suas respectivas repartições em uma floresta amazônica do tipo terra firme. Pretende-se obter as bases para a realização e continuidade de novos experimentos. utilizando-se da hidrologia isotópica.

\section{Material e MÉtodo}

O trabalho foi realizado em área experimental da Bacia Modelo, próxima ao $\mathrm{km} 14$ de estrada ZF-2.

As coletas da água proveniente das chuvas eram feitas semanalmente através de três pluviômetros localizados a céu aberto. Da mistura da água coletada, separava-se uma amostra para análise isotópica.

$\mathrm{Na}$ obtenção das amostras referentes à precipitação interna empregou-se 30 pluv:ômetros localizados no interior da floresta, ao longo de uma linha de aproximadamente 300 metros de comprimento. Da água recolhida em cada pluviômetro, era separada uma porção de cerca de $20 \%$ do volume e da mistura, (") - Trabalho financiado pela Comissão Nacional de Er.ergia Nuclear (CNEN) e International Atomic Energy Agen-
cy (IAEA).

(1) - Faculdade de Ciências Agronômicas de Botucatu, UNESP-SP.

( 2 ) - CENA, Piracicaba-SP.

(3) - Instituto Nacional de Pesquisas da Amazônia, Manaus.AM. 
coletava-se uma amostra para a análise dos conteúdos de deutério e oxigênio-18. SemeIhante procedimento foi seguido na obtenção das amostras provenientes do escoamento pelo tronco, coletadas através de sistemas instalados em 37 árvores.

Para a extração da água do solo utilizouse de metodologia descrita por Reichardt et al. (1977). No interior da floresta, foram insladas 7 estações coletoras sendo que, em cada uma delas, as cápsulas porosas foram colocadas às profundidades de $15,25,50,80 \mathrm{e}$ $120 \mathrm{~cm}$, em relação à superfície do solo. As estações 1 e 2 foram assentadas em terreno relativamente plano e elevado, com o solo classificado texturalmente como argila; as estações 3,4 e 5 localizaram-se em uma encosta, em solo classificado também como argila, enquanto que aquelas de n. 6 e 7 foram instaladas na parte balxa do terreno, em solo clas. sificado como areia-barrenta. O desnível entre a 1." e 7." estação era de $28 \mathrm{~m}$.

$\mathrm{Na}$ Figura 1 mostra-se em esquema, o sistema de extração da água do solo.

O sistema era operado da seguinte forma: com uma bomba de vácuo manual fazia-se vácuo parcial no interior do sistema, fechan do-o até a coleta seguinte. Para retirada da água penetrada através da cápsula, introduzia-se uma sonda ligada a um reservatório coletor, no qual se fazia vácuo parcial, conforme pode ser visto na Figura 1.

De toda água recolhida, em cada sistema, retirava-se uma amostra para análise isotópica.

As coletas de água do igarapé eram feitas junto ao posto fluviométrico, localizado à saída da Bacia Modelo, mergulhando-se um frasco plástico no igarapé.

$\mathrm{Na}$ determinação da relaçāo ${ }^{2} \mathrm{H} /{ }^{\prime} \mathrm{H}$ foi empregada a mesma metodologia de Matsui et al. (1971), enquanto que para o oxigênio-18, o procedimento deu-se de acordo com Matsui (1980).

Os conteúdos de deutério e oxigênio-18 foram expressos na forma de desvio $\left(\delta \%_{\circ}\right)$ definido pela relação:

$$
\delta \%_{\circ}=\left(\frac{a-a p}{a p}\right) \cdot 10^{3}
$$

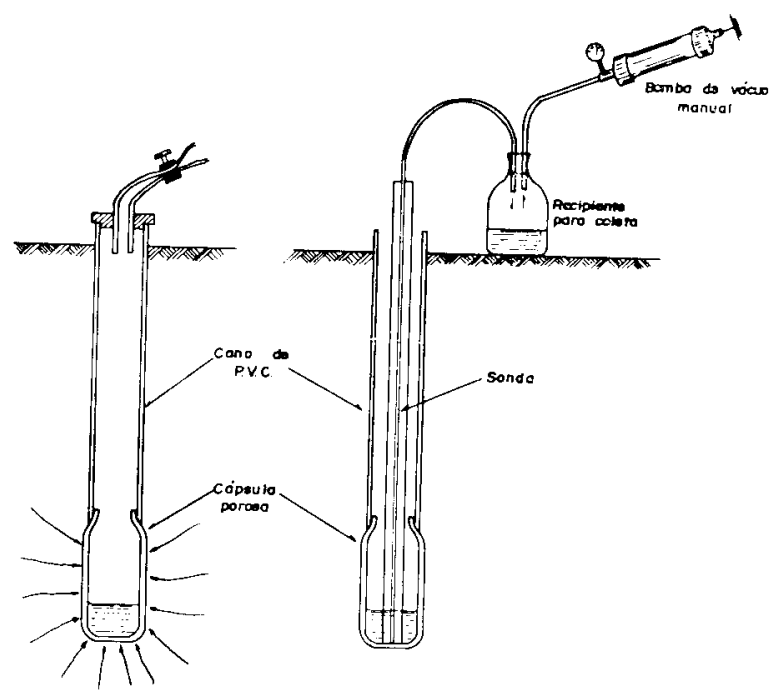

Fig. 1 - Esquema do sistema de extração de água do solo.

onde: "a" é o conteúdo de deutério ou oxigênio-18 da amostra e "ap" é o conteúdo de deutério ou oxigênio-18 da amostra padrão internacional, SMOW (Standard Mean Ocean Water), definida por Craig (1961). Os desvios-padrão nas medidas foram $\pm 1,5 \%$ 。e $\pm 0,2 \%$, para o deutério e oxigênio- 18 respectivamente.

\section{RESULtados}

As variações dos conteúdos de deutério e oxigênio-18, expressos na forma de $\delta^{\%} \%$, são mostradas na Figura 2 para a água de chuva, água de precipitação interna, água escoada pelos troncos, e água do igarapé. Na Figura 3 são apresentadas as curvas de regressão dos valores de $\delta^{2} \mathrm{H}$ em função dos valores obser vados para $\delta^{18} \mathrm{O}$, respectivamente para a água de chuva, água de precipitação interna, água escoada pelos troncos, água do solo e água do igarapé. Com relação a água do solo, determinou-se o valor de $\delta^{2} \mathrm{H}$ apenas para algumas amostras.

As médias ponderadas, verificadas para os conteúdos isotópicos das águas analisadas, estão inclusas na Tabela 1. 

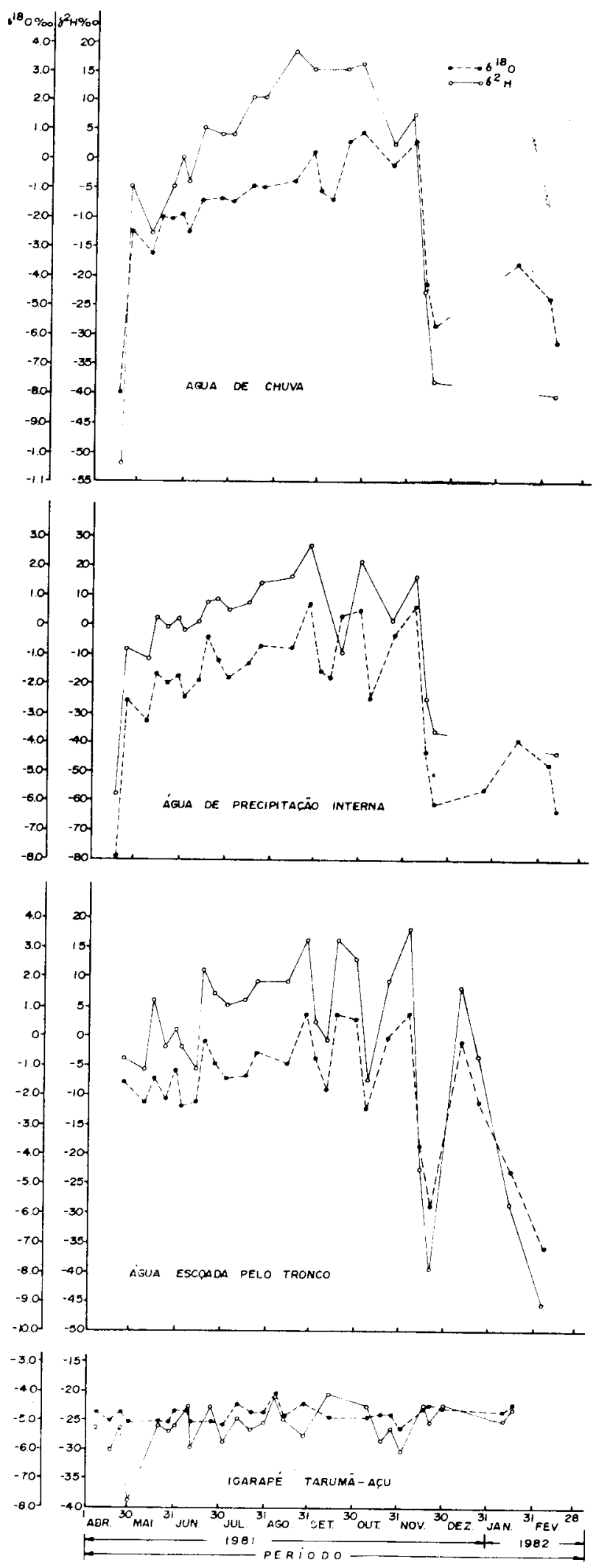

Fig. 2 - Variação de $\delta^{2} \mathrm{H}$ e $\delta^{18} \mathrm{O}$ das águas da chuva, precipitação interna, escoada pelo tronco e do lgarapé Tarumã-Açu em função do período de amostragem.
TABELA 1 - Médias ponderadas de $\delta^{180} \%$ e $\delta^{2} \mathrm{H} \%$ verificadas para as águas analisadas.

\begin{tabular}{lcc}
\hline Agua Analisada & $\delta^{18} \mathrm{O} \%$ & $\delta^{2 \mathrm{H}} \%$ \\
Chuva & $-3,2$ & $-12,4$ \\
Escoada pelos troncos & $-2,3$ & $-8,1$ \\
Precipitação interna & $-3,4$ & $-13,8$ \\
Igarapé & $-4,9$ & $-25,7$ \\
\hline
\end{tabular}

$\mathrm{Na}$ Tabela 2 tem-se os valores dos coeficientes de correlação obtidos entre os valores de $\delta^{18} \mathrm{O}$ da água de precipitação interna e os de $\delta^{18} \mathrm{O}$ da água do solo a $15 \mathrm{cin}$ de profundidade, entre os de $15 \mathrm{~cm}$ e aqueles a $25 \mathrm{~cm}$, c subseqüentemente para as demais profundidades, em função das estações de amostragens.

\section{DISCUSSÃo}

Esta técnica permitiu certas observações que serão úteis na continuidade dos estudos ligados ao ciclo hidrológico da Floresta Amazônica do tipo terra firme.

Com relação aos teores de deutério e oxigênio-18 na água de chuva, os resultados mostrados na Figura 2 indicam uma possivel variação com as estações do ano, sendo mais rica em isótopos estáveis no período que se inicia em fins de abril até início de novembro. Conseqüentemente, as águas representativas da precipitação interna e escoamento pelos troncos, apresentaram variação semelhante, conforme pode ser observado através da Figura 2 .

Para a água do igarapé que drena a Bacia Modelo, essa variação não foi observada e de acordo com os resultados, vistos na Figura 2, os valores de $\delta^{2} \mathrm{H}$, se situaram na faixa de $-20 \%$ a $-30 \%$ enquanto que para o oxigê. nio-18 foram de $-4 \%$ a $-5,5 \%$. Reis et al. (1977) encontraram para a região da ilha de Marajó variações sazonais nos conteúdos de ${ }^{2} \mathrm{H}$ e ${ }^{18} \mathrm{O}$, tanto para a água de chuvas, como em águas superficiais. Observações semeIhantes foram feitas por Salati et al. (1980) para alguns rios da Bacia Amazônica. 

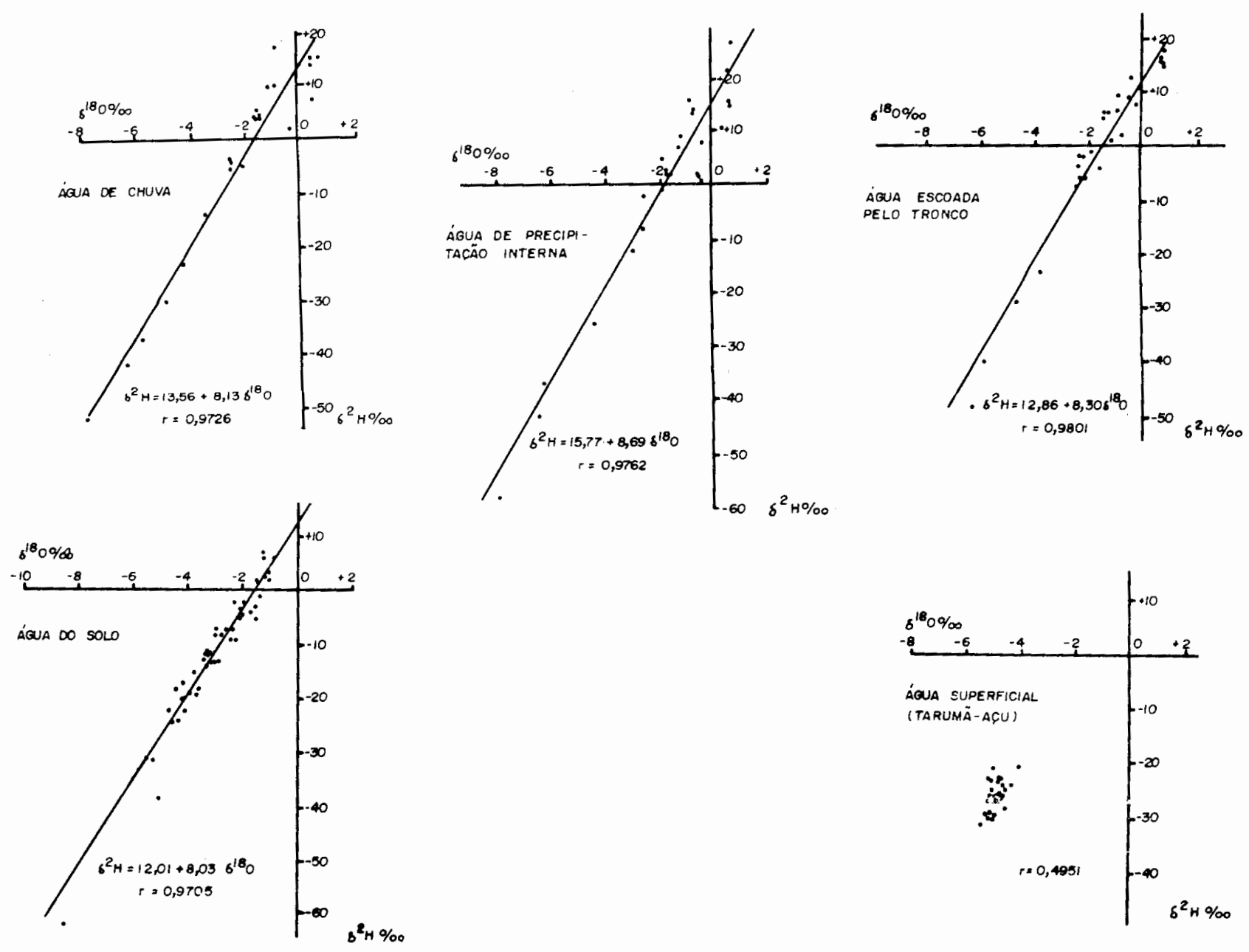

Fig. 3 - Curva de regressão para os valores de $\delta^{2} \mathrm{H}$ em função dos valores de $\delta^{18} \mathrm{O}$ observados para as águas de chuva, precipitação interna, escoada pelo tronco, solo, a diferentes profundidades e superficial.

As pequenas variações nos valores de $\delta^{2} \mathrm{H}$ e $\delta^{18} \mathrm{O}$ da água do igarapé são indícios de que, na altura do lençol freático e franja capilar, ocorre uma grande mistura de águas, bem como um armazenamento de água pelo solo.

Pode ser observado na Figura 3 que a água do igarapé não representou a água de chuva no que se refere as variações de deutério e oxigênio-18.

Verifica-se pela Tabela 1 que as médias ponderadas para a água de chuva e água do igarapé foram para $\delta^{2} \mathrm{H}$ da ordem de $-12,4^{\circ} / c \mathrm{e}$ $-25,4 \%$ respectivamente, enquanto que para o $\delta^{18} \mathrm{O}$ esses valores foram de $-3,2 \%$ e $4,9^{\circ} \%$, mostrando que a água do igarapé comparada. à água de chuva apresentou-se empobrecida, dando indícios de que nas camadas mais pro- fundas do solo ocorreu uma mistura muito grande com águas antecedentes. Por se tratar de um solo de textura fina essas observações são ainda mais acentuadas, conforme já observado por Brinkmann et al. (1963).

O período estudado não inclui parte da estação mais chuvosa, quando os valores isotópicos são mais negativos e que, certamente, levariam as médias ponderadas de $\delta^{2} \mathrm{H}$ e $\delta^{18} \mathrm{O}$, a valores mais negativos.

Comparando-se as equações contidas na Figura 3 , deduz-se que desde a precipitação até a recarga do solo, a água proveniente das chuvas e suas respectivas repartições, não se apresentam fracionadas. Com base nessas observações pode-se deduzir que, embora possa haver uma retenção temporária pelo "litter" 
TABELA 2 - Coeficientes de correlação “ $r$ ” obtidos entre os valores de $\delta^{\mathrm{i}} 0 \%$ da água de precipitação interna e os de $\delta{ }^{18} \mathrm{O} \%$ da água do solo a $15 \mathrm{~cm}$ de profundidade, entre os de $15 \mathrm{~cm}$ e aqueles a $25 \mathrm{~cm}$,e subseqüentemente para as demais profundidades, em função das estações de amostragens.

\begin{tabular}{|c|c|c|c|c|c|}
\hline Estações & $\begin{array}{l}r \\
\mathrm{Pl} \times 15\end{array}$ & $\begin{array}{l}r \\
15 \times 25\end{array}$ & $\begin{array}{l}r \\
25 \times 50\end{array}$ & $\begin{array}{l}r \\
50 \times 80\end{array}$ & $\begin{array}{l}r \\
80-120\end{array}$ \\
\hline 01 & $\begin{array}{l}0,7602^{*} \\
n=12\end{array}$ & $\begin{array}{l}0,7375^{*} \\
n=13\end{array}$ & $\begin{array}{l}0,6861^{*} \\
n=12\end{array}$ & $\begin{array}{l}0,4360(n s) \\
n=15\end{array}$ & $\begin{array}{l}0,6929^{*} \\
n=16\end{array}$ \\
\hline 02 & $\begin{array}{l}0,7543^{\circ *} \\
n=13\end{array}$ & $\begin{array}{l}0,9080^{*} \\
n=16\end{array}$ & $\begin{array}{l}0,6447^{* *} \\
\mathrm{n}=15\end{array}$ & $\begin{array}{l}0,8375^{* *} \\
n=19\end{array}$ & $\begin{array}{l}0,5682^{*} \\
\mathbf{n}=17\end{array}$ \\
\hline 03 & $\begin{array}{l}0,7041^{* *} \\
n=20\end{array}$ & $\begin{array}{l}0,8656^{* * *} \\
n=21\end{array}$ & $\begin{array}{l}0,3927(\mathrm{~ns}) \\
\mathrm{n}=19\end{array}$ & $\begin{array}{l}0,3952(\mathrm{~ns}) \\
\mathrm{n}=19\end{array}$ & $\begin{array}{l}0,4825^{\star} \\
n=21\end{array}$ \\
\hline 04 & $\begin{array}{l}0.5557^{*} \\
n=19\end{array}$ & $\begin{array}{l}0,8895^{* *} \\
n=22\end{array}$ & $\begin{array}{l}0,8881^{* *} \\
n=24\end{array}$ & $\begin{array}{l}0,4891^{*} \\
n=24\end{array}$ & $\begin{array}{l}0,6549^{* * *} \\
n=29\end{array}$ \\
\hline 05 & $\begin{array}{l}0,7639^{* *} \\
n=14\end{array}$ & $\begin{array}{l}0,7308^{* *} \\
n=12\end{array}$ & $\begin{array}{l}0,6578^{\circ} \\
n=13\end{array}$ & $\begin{array}{l}0,7986^{*} \\
n=16\end{array}$ & $\begin{array}{l}0,5631^{*} \\
n=15\end{array}$ \\
\hline 06 & $\begin{array}{l}0,9067^{* \ldots} \\
n=14\end{array}$ & $\begin{array}{l}0,9503 * * \\
n=12\end{array}$ & $\begin{array}{l}0,9038 * * * \\
n=23\end{array}$ & $\begin{array}{l}0,7603^{* *} \\
\mathrm{n}=25\end{array}$ & $\begin{array}{l}0,6778^{* * *} \\
n=27\end{array}$ \\
\hline 07 & $\begin{array}{l}0,9437^{*} \\
n=12\end{array}$ & $\begin{array}{l}0,9629^{* *} \\
\mathrm{n}=11\end{array}$ & $\begin{array}{l}0,9238^{* * *} \\
n=10\end{array}$ & $\begin{array}{l}0,3661(\mathrm{~ns}) \\
\mathrm{n}=14\end{array}$ & $\begin{array}{l}0,5935^{*} \\
n=12\end{array}$ \\
\hline
\end{tabular}

$(*)$ - Significotivo do nivel de $5 \%$.

$\left({ }^{* *}\right)$ - Idem, a $1 \%$.

$\left({ }^{* *}\right)$ - idem, o $0,1 \%$

(n ) - Nọ de dados.

antes da água penetrar o solo, a evaporação ćireta da água do solo sob cobertura florestal deve assumir proporções insignificantes quando comparada a outros tipos de perdas. Jordan \& Heuveldop (1981) observaram também, por metodologia diferente, que a taxa de perda de água do solo por evaporação sob cobertura florestal assume valores despreziveis. As médias ponderadas, relativas à água de chuva e da precipitação interna, foram semelhantes, mas diferiram daquelas obtidas para a água escoada pelos troncos, cujas médias foram iguais a $-8,1 \%$ e $-2,3 \%$ para $\delta^{2} \mathrm{H}$ e $\delta^{18} \mathrm{O}$, respectivamente.

Tais valores demonstraram que a massa de água escoada através dos ramos, galhos e troncos foi enriquecida em isótopos estáveis, em relação à água de chuva, cujo enriquecimento se deu, provavelmente, em função do processo de evaporação. Esses resultados vêm confirmar que, o escoamento pelo tronco representa uma parcela muito pequena da precipitação total, provavelmente, em razão da morfologia das plantas, bern como, em função de um escoamento lento da água, que permite uma maior taxa de perda por evaporação, antes de atingir o solo.

Com relação a água do solo, os valores do coeficiente de correlação indicados na Tabela 2 dão a entender que, para as camadas superficiais de 0 a $25 \mathrm{~cm}$ essa água é bem caracterizada em termos de oxigênio-18 demonstrando que, nesse perfil, praticamente não ocorre misturas com águas antecedentes.

Dessa observação deduz-se que o tempo de residêricia da água, no referido perfil, é relativamente pequeno, sendo removida para perfis mais profundos ou pelas plantas através do processo de transpiração. Para o solo classificado como areia barrenta, esiações 6 e 7, essa camada se estende até a profundidade de $50 \mathrm{~cm}$.

Nas demais camadas os valores de correlação decrescem indicando uma mistura de água que se move através do perfil, com águas 
antecedentes. Pode ser visto que para o solo texturalmente classificado como areia barrenta, essas observações são mais acentuadas. Brinkmann et al. (1963) e Zimmermann, et al. (1966) trabalhando com solo arenoso, observaram variações marcantes em deutério na água do solo em função das variações desse elemento na água de chuva.

Acredita-se que os valores de $r_{\mathrm{Pl}} \times 15$ das estações 3 e 4 apresentaram-se relativamente inferiores aos demais $r_{\mathrm{PI}} \times 15 \mathrm{em}$ razão de um possível escoamento sub-superficial, da estação 1 e 2 em direção às camadas superficiais das estações 3 e 4 .

Para as estações localizadas na parte mais alta do terrenc, estações 1 e 2, respostas aos valores de $\delta^{18} \mathrm{O}$ das águas de precipitação interna é da ordem de uma semana para os pontos mais superficiais, 15 e $25 \mathrm{~cm}$. Verificou-se que para uma precipitação interna com um valor de $\delta^{18} \mathrm{O}$ igual a $-7,9 \%$ ocorrida na semana de $11 / 04 / 80$ a $18 / 04 / 80$, os valores de $\delta^{18} \mathrm{O}$ da água do solo, a $15 \mathrm{~cm}$ e $25 \mathrm{~cm}$ de profundidade foram da ordem de $-6,9 \%$ o $-5,8 \%$ respectivamente, para a estação 2 . Na semana seguinte, como a precipitação interna teve um $\delta^{18} \mathrm{O}$ da ordem de $-2,6 \%$ aqueles valores passaram para $-4,8 \%$ e $-5,7 \%$. Para as profundidades maiores, muito embora o valor de $-7,9 \%$ obtido para a água de precipitação interna tenha sido diluído pela mistura com águas antecedentes, observou-se que a massa líquida caracterizada por esse referido valor, levou cerca de 3 semanas para uma mistura com a água residente no ponto situado a $80 \mathrm{~cm}$ da estação 1 , quando o valor de $\delta^{18} \mathrm{O}$ passou de $-3,9 \%$ para $-5,2 \%$. Da mesma maneira, semelhante comportamento foi verificado para a estação 2 .

Os valores de $\delta^{18} \mathrm{O}$ observados para as estações 3 e 4, apresentaram-se com uma amplitude de variação inferior àquelas obtidas para as demais estações de amostragens. Essa observação vem reforçar outra anteriormente feita, na qual supóem-se a existência de um fluxo sub-superficial da estação 1 e 2 , na profundidade de 50 ou $80 \mathrm{~cm}$, em direção aos pontos superficiais junto as estações 3 e 4 .
Para as estações 6 e 7 , situadas na parte baixa do perfil, em solo classificado texturalmente como areia barrenta, as variações observadas para os valores de $\delta^{18} \mathrm{O}$ da água do solo em função daqueles da água de precipitação interna foram mais acentuadas que para as demais estações. Observou-se que para um valor de $-7,9 \%$ da água de precipitação interna verificado na semana de 11 a 18/04/80, a água do solo apresentou respostas relativamente imediatas para os pontos a 15 e $25 \mathrm{~cm}$ de profundidade, com vaiores de $-7,1 \%$ e $7,4 \%$ respectivamente, na estação 6 e $-8,3 \%$ e $\cdot 7,7 \%$ para a estação 7 . Ainda de acordo com os dados obtidos, deduz-se que a massa de água caracterizada por um $\delta^{18} \mathrm{O}$ igual a $-7,9 \%$ levou, de um modo geral, 2 semanas para uma mistura com a água situada a profundidade de $50 \mathrm{~cm}, 3$ semanas para a profundidade de $80 \mathrm{~cm}$ e cerca de 5 semanas para os pontos situados a $120 \mathrm{~cm}$ da superfície do solo.

Semelhantes comportamentos para a água do solo foram descritos por Brinkmann et al. (1963), Zimmermann et al. (1966) e Vandor et al. (1977), diferindo, no entanto, em termos de ordem de grandeza.

Pretende-se, com os resultados ora obtidos determinar o tempo de residência da água no solo através de modelo apropriado ao caso, bem como efetuar-se o balanço isotópico das massas de água envolvidas nas diversas fases, com o que pode-se estimar a percentagem da água de chuva correspondente à evapotranspiração do ecossistema em questão.

Esses pontos, no entanto, serão objetos de trabalhos a serem apresentados posteriormente ao que ora é exposto.

\section{Agradecimentos}

Os autores agradecem à Comissão Nac:onal de Energia Nuclear (CNEN) e a Agência Internacional de Energia Atômica (IAEA) pelo apoio recebido na realização deste trabalho, através de recursos financeiros. 


\section{SUMMARY}

It was possible to observe the rain water values of $\delta^{2} \mathrm{H}$ e $\delta^{18} \mathrm{O}$ and theirs respective repartition, including the soil water until the depth of $1.20 \mathrm{~m}$. The work was done in the Model Basin in an experimental area near the $\mathrm{Km} 14$ of the $\mathrm{ZF}-2$ road. The obtained results showed that the isotopic composition of the rain water vary with the season of the year, and are more euriched in ${ }^{2} \mathrm{H}$ and ${ }^{18} \mathrm{O}$ in the period wich begins at the end of April and beginning of November. These variations were not observed in the igarapé Tarumã-Açu water, suggesting a blend of waters and a high storage of water by the soil.

\section{REFERENCIAS BIBLIOGRAFICAS}

BRINKMANN, R.; EICHLER, R.; EHHALT, D.; MUNNICH, K.O.

1963 - Über den deuterium gehalt von Niederschlage und Crundwasser. Naturwissenschaften, 19: 611 .

CRAIG, $\mathrm{H}$.

1961 - Standar for reporting concentrations of deuterium and oxigen-18 in natural waters. Science, 133 (3467): 1833-1834.

DALL'OLIO, A.

1976 - A composição isotópica das precipitações do Brasil: modelos isotérmicos e a influência da evapotranspiração na Bacia Amazônica. Dissertação de Mestrado, ESALQ, Piracicaba, 180p.

DALL'OLIO, A.; SALATI, E.; AZEVEDO, C.T.; MATSUI, E. 1979 - Modelo de fracionamento isotópico da água na Bacia Amazônica. Acta Amazonica, 9 (4): $675-687$.

GONÇALVES, A.R.L.

1979 - Determinação do tempo de residência da água de chuva em algumas bacias hidrográficas através de valores de isótopos. Dissertação de Mestrado. ESALQ, Piracicaba, 106p.

JORDAN, C.F. \& HEUVELDOP, J.

1980 - The water budget of an Amazonia rain forest. Acta Amazonica, 11 (1): 87-92.

MATSUI, E.

1980 - A simple method using a disposable syringe to prepare samples for $\delta^{18} \mathrm{O}$ measurements in water samples. Anal. Chem. Acta (no prelo).
MATSUI, E.; SALATI, E.; FERRAZ, E.S.B.

1971 - Medida da variação natural da relação $\mathrm{D} / \mathrm{H}$ em amostras de água. CENA, Boletim Científico (1): 31 .

MATSUI, E.; SALATI. E.; BRINKMANN, W.L.F.;

FRIEDMAN, I.

1972 - Vazões relativas dos rios Negro e Solimões através das concentrações de ${ }^{180} \mathrm{O}$. Acta Amazonica, 3 (2): 3i-46.

MATSUI, E.; SALATI, E.; FRIEDMAN, I.;

BRINKMANN, W.L.F.

1976 - Isotopic hydrology in the Amazonia 2. Relative discharges of the Negro and Solimões rivers through. ${ }^{18} \mathrm{O}$ concentrations. Water Resources Researc, 12 (4): 781-785.

REICHARDT, K.; LIBARDI, P.L.; MEIRELLES, N.M.F.;

FERREIRA, F.F.H.; ZAGATO, E.A.G.; MATSUI, E.

1977 - Extração e análise de nitratos em solução do solo. Rev. Bras. Ci. Solo, 1: 130-132.

REIS, C.M.; TANCREDI, A.C.N.S.; MATSUI, E. \&

SALATI, E.

1977 - Caracterização das águas da região de Marajó através de concentrações de ${ }^{18} \mathrm{O}$ e $\mathrm{D}$. Acta Amazonica, 7 (2): 209-222.

SALATI, E.; MARQUES, J.; MOLION, L.C.B.

1978 - Origem e distribuição das chuvas na Amazônia. Interciência, 3 (4): 200-205.

SALATI, E.; DALL'OLIO, A.; MATSUI, E.; GAT, J.

1979 - Recycling of water in the Amazon basin: an isotopic study. Water Resources Research, 15 (5): 1250-1258.

SALATI, E.; MATSUI, E.; CERVELLINI, A.; FRIEDMAN, 1. 1980 - Isotope hydrology in Amazonia. Part I: $\delta D$ in river waters. Rev. Energia Nuclear e Agricultura, 2 (2): 101-132.

VANDOR, H.; FRITZ, P.; GILLHAM, R.W.; BROWN, R.M. 1977 - Environmental isotope studies of the infiltration process. Atomic Energy of Canada, Chalk River, Ontario. p. 56-61.

ZIMMERMANN, V.; EHHALT, D.; MUNNICH, K.O.

1966 - Soil water movement and evapotranspiration; changes in the isotopic composition of the water. In: Symposium of Isotopes in Hydrology. IAEA, Vienna, 14 a 18 de novembro de 1966.

(Aceito para publicação em 28/04/82) 